\title{
The prevention and management of the coronavirus disease 2019 (COVID-19) outbreak in radiology departments in epidemic areas
}

\author{
Ming Deng ${ }^{1}$ \\ Received: 30 March 2020 / Accepted: 7 April 2020 / Published online: 18 April 2020 \\ (c) Japan Radiological Society 2020
}

\begin{abstract}
This review based on the actual results of epidemic prevention management in radiology departments in epidemic areas, and using designated hospital management methods, to summarize the radiology protection system. With the cooperation of the whole hospital and radiology department, a number of effective evaluation methods have been carried out to ensure the optimization of clinical protection. These practical methods provide a basis for the further promotion of management strategies and reduction of nosocomial infection. To our knowledge, the establishment of standardized protection and clear process has opened up a new idea of epidemic prevention management, which can effectively provide timely, efficient, and accurate support for clinical research, and promote the transformation from clinical research type to scientific research management type.
\end{abstract}

Keywords Novel coronavirus · Pneumonia epidemic Prevention · Hospital management

\section{Background and management introduction}

Starting in December 2019, many patients contracted fever from unknown causes in Wuhan, and the infection can spread by human-to-human transmission [1]. This is a new type of infectious disease with an extremely high transmission rate. The virus now known to be the cause is named severe acute respiratory syndrome coronavirus 2 (SARSCoV-2). It has extremely high infectivity, a short doubling time, a basic reproduction number of 2.2 , and a high risk of nosocomial infection. There have been 81,134 confirmed cases in China, with 67,799 confirmed cases in Hubei province, including 50,004 cases in Wuhan, with 2480 deaths.

A total of 20,349 computed tomography (CT) scans have been completed in our hospital. According to the "Novel Coronavirus Pneumonia Diagnosis and Treatment Plan (Provisional 7th Edition)" issued by the National Health Commission of the People's Republic of China [2], patients need to undergo high-resolution CT (HRCT) test to help diagnose the disease. HRCT not only can display the lesion

Ming Deng

dr_dengming@whu.edu.cn

1 Department of Radiology, Zhongnan Hospital of Wuhan University, Wuhan University, \#169 Donghu Road, Wuchang District, Wuhan City 430071, Hubei Province, China at the millimeter scale, but also can accurately quantify the scope and extent of the lesion. Therefore, CT examination is particularly important for the phased evaluation of coronavirus disease 2019 (COVID-19), while HRCT has become the main source of evidence for clinical diagnosis of COVID-19 [3].

However, due to the large number of patients in the epidemic area, there is a relative shortage of diagnosis and treatment resources in the radiology departments, which lead to many suspected and uninfected patients receiving HRCT examinations in a concentrated area and time. At the same time, at the initial stage of the disease outbreak, there were many patients with confirmed COVID-19, suspected patients, hospitalized emergency patients, and medical staff working in isolation wards, resulting in a high risk and probability of nosocomial infection. Therefore, how to avoid cross-infections in hospitals, prevent and control the spread of SARS-CoV-2 between doctor and patient, and protect the health and safety of healthy people and front-line medical and nursing staff is the current problem that the radiology departments face [4].

At present, international reports indicate that the number of confirmed cases of COVID-19 has begun to increase in many countries around the world, especially Italy, Iran, Spain, South Korea, Germany, and French. This paper lays out the plan for disease prevention and control in the 
radiology department of our hospital to provide a reference for hospital managers and our peers in radiology departments around the world.

\section{Epidemic theory updates and leaders for special tasks}

\section{Establishing a COVID-19 leadership team}

Cooperating with the hospital leadership and infectious disease department, we set up the corresponding group leader, quality control personnel, liaison personnel, and emergency response personnel. The head of the radiology department acted as the group leader to conduct unified command and coordination and assigned 1-2 professionals to manage the working area and daily-use equipment in turns to ensure that the working environment and the working equipment area met the environmental standards, while cooperating with various managers in the hospital. In the meantime, because of the high rate of use and consumption of medical goods and materials, special management personnel should also be appointed to ensure that the protection of front-line medical personnel meets the standard.

\section{Nosocomial infection awareness training: continuous updating and learning of COVID-19 diagnosis and treatment criteria}

To prevent the spread of COVID-19 caused by patients having delayed treatment because of failure of timely diagnosis, in the 1st-5th versions of the "Novel Coronavirus Pneumonia Diagnosis and Treatment Plan", clinically diagnosed cases were gradually included in the diagnosis criteria of the severe epidemic area (Hubei Province), and the suspected cases with typical imaging characteristics of COVID-19 can be clinically diagnosed as COVID-19 and treated immediately. Based on this major adjustment, chest HRCT is the preferred imaging method for COVID-19. The learning of different versions of updated diagnosis and treatment guidelines, nosocomial COVID-19 diagnosis and treatment processes, and nosocomial infection prevention should always be implemented in the full process of COVID-19 diagnosis and treatment. All radiology department staff should be familiar with the clinical manifestations, imaging features, and latest developments on COVID-19. We invited experts from the infectious disease department to train our staff on prevention and protection and to instruct them about precautions. We utilized online platforms to update the latest prevention and protection strategies and instructing standards in real time.

\section{Fully grasping the principles of prevention and treatment of infectious diseases and disinfection management measures}

To enhance the awareness of prevention and disinfection management, we invited many hospital nosocomial infection experts to our site for prevention training and recorded instructional videos. In the training for the radiology department, the experts emphasized training the technicians on how to conduct self-protection and environment disinfection while performing the scanning. We required everyone to understand the material, practice the procedures, and pass a test. The technicians could only come back to work after the videotape of their performance had been reviewed and met the requirements. In the specific implementation process, a journal was set up, and the disinfection status was strictly registered and reported by taking photographs every day. The test was scored out of 100 points. The staff had to get a full score, including 40 points for personal protection. Personal protection included seven steps: hand washing (alcohol disinfection), standard wearing of masks and caps, putting on and removing protective clothing (isolation gear), wearing of gloves and goggles, familiarity with the three areas (sterile area, contaminated area, and semi-contaminated area) and two pathways (medical personnel pathway and patient pathway), and CT diagnosis procedures. The score was evaluated based on the order of precedence and the degree of meeting operational specifications and operational proficiency. The standards and distribution of scores for disinfection of the objects, equipment, air, and floor of the examination room are shown in Table 1.

\section{Personal protection levels and precautions}

General protection Applicable to hospital emergency patients and physical examiners: wear work uniform, disposable medical mask, and, when necessary, gloves.

Level 1 protection Applicable to patients with fever, the outpatient medical staff who conduct prescreening and triage, and staff who work in the fever clinic or infectious disease clinic: wear a disposable work cap, a disposable medical mask (those having contact with patients with infectious disease history need to wear an N95 respirator), a work uniform, isolation clothing (wear disposable isolation clothing when doing prescreening and triage), and disposable latex gloves when necessary, and strictly follow hand hygiene practices.

Level 2 protection Applicable to medical personnel in close contact with and involved in the diagnosis and treatment of suspected or confirmed patients: wear a disposable 
Table 1 Disinfection methods and evaluation scores

\begin{tabular}{|c|c|c|c|c|}
\hline Item & Disinfectant (equipment) & Frequency/time & Emergency spill treatment & Full score \\
\hline Object disinfection & $1000 \mathrm{mg} / \mathrm{L}$ chlorine-containing disinfectant ${ }^{\mathrm{a}}$ & Once/4 h & \multirow[b]{2}{*}{$\begin{array}{l}\text { After cleaning with disposable, soft, } \\
\text { absorbent material with disinfectant, } \\
\text { wipe the object using a cloth soaked with } \\
2000 \mathrm{mg} / \mathrm{L} \text { chlorine-containing disinfect- } \\
\text { ant }\end{array}$} & 20 \\
\hline Equipment disinfection & $2000 \mathrm{mg} / \mathrm{L}$ chlorine-containing disinfectant $\mathrm{a}^{\mathrm{a}}$ & Twice/day & & 20 \\
\hline Air disinfection & Air sterilizer or ultraviolet lamp & 4 times/day & $\begin{array}{l}\text { Air disinfection }>30 \text { min, ultraviolet dis- } \\
\text { infection }>60 \text { min and close the internal } \\
\text { shielding door }\end{array}$ & 10 \\
\hline Ground disinfection & $1000 \mathrm{mg} / \mathrm{L}$ chlorine-containing disinfectant ${ }^{\mathrm{a}}$ & Once/4 h & Timely routine disinfection treatment & 10 \\
\hline
\end{tabular}

${ }^{\mathrm{a} C}$ Corrosion-resistant objects and equipment are wiped twice with $75 \%$ ethanol

work cap, protective goggles or a face mask (anti-fog type), a medical protective mask, protective clothing or isolation clothing, disposable latex gloves, and disposable shoe covers, and strictly follow hand hygiene practices.

Level 3 protection Applicable to aerosol operators for suspected or confirmed patients: wear a disposable working cap, a medical protective mask, protective goggles or a protective face shield (or a comprehensive respirator or a positive-pressure head cover), protective clothing, disposable latex gloves, and disposable shoe covers, and strictly follow hand hygiene practices.

Doctors, medical technicians, and nurses, in addition to their personal protection, need to pay special attention to fire safety. In the hospital, there are many flammable materials, flammable chemical reagents, pressure vessels, and electric heating machines. If these are not properly managed, it is easy for an accidental fire or explosion to occur. Check the room temperature and humidity to make sure that they are at appropriate levels. After disinfecting with alcohol, sparks should be strictly avoided. At the same time, it is necessary to set up a one-button alarm device in a conspicuous position, as well as to post the phone numbers for the fire, rescue, emergency, and security patrols. Every day, it is necessary to check that the emergency exits are not blocked, the signs are clear, the fire facilities are in order, and the emergency response plan is complete.

\section{Plan the inspection procedures and different types of inspection areas}

Before examination of the patients, the radiology department focused on transforming the corresponding equipment rooms and examination rooms in strict accordance with the three areas and two pathways, to establish the activity areas for the patients and medical staff, including the contaminated area, semi-contaminated area, and clean area. Oneway flow should be implemented for the registrar, operator, technician, and disinfection and cleaning personnel who have direct contact with the patients to avoid cross-infection.
At the same time, personnel should be appointed to conduct thorough disinfection of the examination area, equipment, and pedestrian passageways, and CT equipment room and examination room should be disinfected for $24 \mathrm{~h}$ without interruption. Any rooms that do not meet these standards shall be remodeled to ensure that there will be no gaps in coverage.

At the same time, to prevent cross-infection, it is necessary to strictly divide and disinfect the registration room, the injection room, offices of technicians and doctors, consultation room, and public waiting areas. The staff of the department should go to and from work through a restricted passageway that uses access control and does not permit entry or exit by other persons. Technicians should put on protective equipment in the clean area and remove protective equipment in the buffer zone. Technicians in contaminated areas are not permitted to enter a clean area before their shift ends. The shifts run 4-6 h. When changing shifts, according to the procedure, technicians need to remove protective clothing in the buffer zone and get disinfected first before entering the clean area. In addition, technicians should set up monitoring equipment and voice transmission systems (calling systems) in and out of the operating room to react to emergencies at any time.

\section{Material management and equipment support}

\section{Protective material management}

Strictly distinguish the first-line and second-line personnel on duty, and make reasonable arrangement of staff by levels of seniority. Before the daily shift, protective equipment, N95 masks, protective eyepieces, caps, gloves, shoe covers, and protective clothing should be distributed according to infection classification. The principles of protection optimization and resource maximization should be followed to ensure the use of protective equipment for numerous 
personnel in contact with patients. At the same time, the material management shall be reported to the corresponding team leader to ensure a smooth shift change. Off-duty personnel should handle the waste protective equipment and sterilize the protective equipment that can be reused to ensure that all materials are in a clean state. The whole process should be recorded and signed off/on.

\section{Computer management of equipment and simplified examination process}

To cope with the treatment of many COVID-19 patients and diagnosis of suspected patients, we first evaluated the performance and maximum capacity of the equipment. As far as possible, we used a special CT equipment with strong performance, which could meet the examination requirements of a 400-person daily load. The equipment is only used for examination of patients with fever to minimize cross-infection. At the same time, to control the movement of sources of infection in the hospital, we selected and cooperated with the fever clinic to arrange an examination green path to the nearest equipment to ensure the smooth $\mathrm{CT}$ examination of patients and reduce the waiting time in the examination and the time to find the examination equipment. Second, CT equipment should support the automatic upload function of examination images. After a patient examination, all the examination information is automatically subjected to thinslice reconstruction and uploaded to the Picture Archiving and Communication System for radiologists to read the film in time (Fig. 1).

Before CT examination, the diagnosing physician should make electronic labels, set up a reminder pop-up, and save alternate contact information in HIS for confirmed and suspected COVID-19 cases. When the radiology department receives the HRCT examination information from the patients, designated personnel send an individualized reminder according to the patient's condition, examination requirements, and nosocomial infection status and call the patients to inform them of the matters needing attention. When there are too many patients, diagnosis videos will be played on a loop. Patients should wear masks and disinfect their hands quickly before HRCT examination. We do not recommend queuing and set up waiting areas. The patient should understand the $\mathrm{CT}$ examination procedure to the greatest extent they can. Therefore, a noticeable prompt or voice prompt should be set in the fever clinic and next to the CT examination room. Metal accessories worn by the patients should be removed in advance, and the patients should hold their breath during the examination to ensure the success of the examination.

After the patient finishes the examination, his or her registered mobile phone will receive a webpage link and an electronic QR code so that the patient can check the electronic report and high-definition image at any time. The hospital information system can also check the patient's examination report and image information simultaneously. The whole process is computerized, paperless, and convenient.

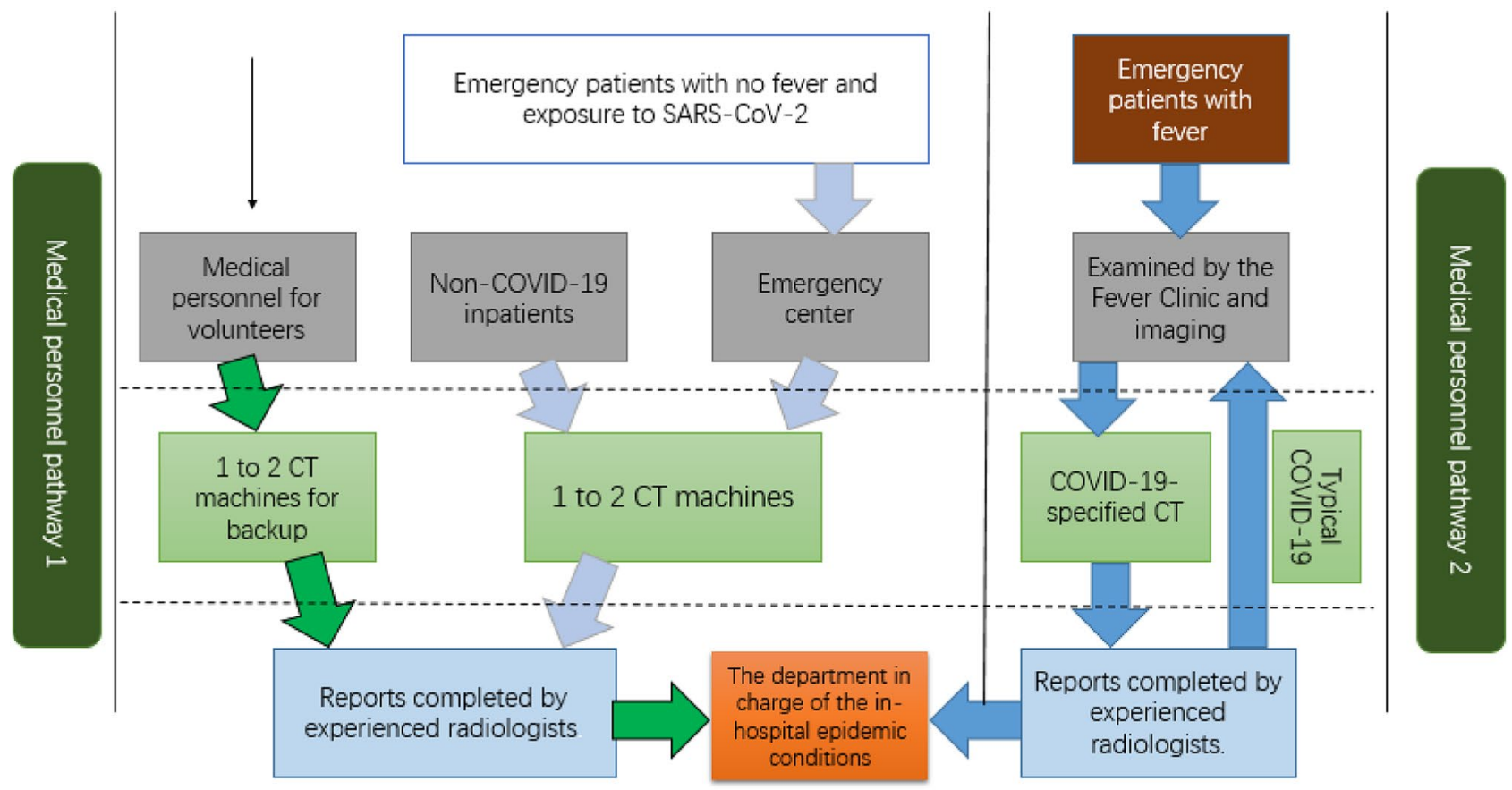

Fig. 1 Flowchart of radiology and imaging work during the epidemic period 
After the technician completes the examination, the image is automatically uploaded to the doctor's workstation, and the radiologist quickly completes the diagnosis report based on the patient's image data to ensure that the patient can get the report within 30 min after the examination. The radiologist should be separate from patient access and examination equipment during the report-writing process. Radiologists should complete the examination report as soon as possible and objectively. During the process, they can refer to artificial intelligence tools (such as medical diagnosis based on images, YITU Healthcare Technology Co., Ltd., Hangzhou, Zhejiang) to quantitatively or semi-quantitatively record the distribution of different lesions, their volume ratios, and their involvement of the lung segments. Valid information reported by physicians will be automatically updated to the HIS system for storage. Patients can obtain reports and high-definition images through mobile electronic terminals, as well as print physical film and paper reports at self-service printing terminals.

\section{Technical route and examination parameter planning}

\section{Developing special equipment for different inspectors}

The department should identify different populations seeking treatment, including patients with fever of unknown causes, highly suspected COVID-19 patients, and confirmed COVID-19 patients, and their physical examination should be done on designated CT equipment. To reduce nosocomial infection, the routine physical examination of patients in the hospital and non-COVID-19 inpatients should not be done on the above equipment.

\section{Reasonable collocation of personnel with different professional titles, nature of work, and experiences to ensure smooth coordination of work}

In the face of many patients seeking medical treatment, we collocate doctors, technicians, and nurses by their work experience to ensure quick responses and proper handling of emergencies. In particular, when the staff with frontline experience in fighting against pneumonia epidemics is teamed up with those without relevant work experience, the team coordination efficiency will be significantly enhanced. Especially in the early stage of the COVID-19 outbreak, this helped the junior staff to overcome their fears.

\section{Patient examination}

After the patient is ready, the operating technician keeps a certain distance $(>1 \mathrm{~m})$ whenever possible throughout the whole process. Staff should try to use their voice to interact with the patient as far as possible to reduce direct contact, and monitoring equipment should be reasonably configured to help with automatic bed lifting in the operation room to minimize contact. After the examination of COVID-19 patients or suspected patients, the technician informs patients of the preliminary results according to the images. If no pulmonary infection occurs, the patient leaves the examination room as soon as possible and goes to the self-help printer to obtain the film and report at the appointed time (or the patient can obtain the results at their own convenience). Any suspected pulmonary infection should be reported to the medical affairs office and fever clinic immediately (the diagnostic physician should complete the report within $30 \mathrm{~min}$ ). Immediately after the CT examination of the COVID-19 patient, the cleaners should be instructed to disinfect according to the level 1 protection requirements, disinfect the examination bed surface and the floor, and replace the CT examination bed sheets. Moreover, disinfection of the air, floor, and equipment should be conducted at the same time(s) every day (see Table 1 for the standard).

\section{Adult pulmonary HRCT parameters}

Patients are placed in a routine supine position, with the body placed in the middle of the examination bed, with the head resting on both arms. If the patient has difficulty raising the arms, both arms can be placed at the sides of the body. The CT adopts an axial spiral scan, ranging from the apical of the lung to the lower margin of the bilateral costal diaphragmatic angle. The scanning field is $40 \mathrm{~cm}$, conventional $100 \mathrm{kV}, 20 \mathrm{~mA}$, automatic tube voltage [tube voltage $100 \mathrm{kV}$ for patients with body mass index (BMI) $<25 \mathrm{~kg} / \mathrm{m}^{2}$; tube voltage $120 \mathrm{kV}$ for BMI $\geq 25 \mathrm{~kg} / \mathrm{m}^{2}$ ], automatic tube current (range 100-350 mAs) modulation system. The acquisition slice thickness is $5 \mathrm{~mm}$; the reconstruction slice thickness and the reconstruction inter-slice gap are $1.25 \mathrm{~mm}$; the tube rotation time is $0.6 \mathrm{~s} / \mathrm{r}$; the pitch is 1.2 ; the iterative reconstruction technology is started. Conventional image reconstruction: the lung window image (window width $1500 \mathrm{HU}$ and window level-700) and mediastinal window image (mediastinum window: $200 \mathrm{HU}$ and window level $40 \mathrm{HU}$ ) were reconstructed with slice thicknesses of $5 \mathrm{~mm}$ and $1 \mathrm{~mm}$, respectively. 


\section{Humanistic care and green path management}

COVID-19 is a sudden disaster that has resulted in a public crisis. To overcome this crisis, it is necessary to show humanistic care and a care for life with the greatest emotional support and spiritual cohesion. During the beginning of the epidemic, the lives and safety of the front-line doctors, technicians, and nurses were threatened, and the normal life and work schedules were seriously disrupted. Our director and senior administrators often come to the front lines, eat with the staff, work with them, face the epidemic together, and thereby boost the morale of each staff member. Second, on a personal level, we have organized a step-by-step process to purchase staff essential goods, and have coordinated and made unified arrangements, including the reservation of boxed meal packages, the supply of boiled water, and the planning of work routes. For employees with travel difficulties, we uniformly organize department staff, team up in groups, coordinate transportation, ensure smooth commuting, avoid travel difficulties due to traffic control (which will affect their normal work), and avoid taking public transportation (which increases the risk of infection).

In addition, we timely announce the health status of the employees every day, isolate and treat the SARS-CoV2-infected employees, and provide necessary help, including daily disinfection supplies, masks, and other supplies, to ensure that their family members are at a minimal risk of infection, as well as providing necessary psychological care. We also provide timely assistance to employees and family members who have an infection secondary to SARSCoV-2 infection and effectively solve their practical difficulties in living and seeking medical treatment. At the same time, volunteers from our department will buy and deliver daily necessities to the elderly for whom it is inconvenient to travel or are isolated at home. We also provide for the needs of retired experts, delivering commonly used medicines and necessities to express our care and warmth toward them.

A special care should be given to special groups of patients, including pregnant and postpartum women and children. So far, our hospital has received 176 pregnant women and 21 children, all of whom underwent CT examination and nucleic acid amplification test by reverse transcriptionpolymerase chain reaction (RT-PCR) to detect SARS-CoV-2 in the hospital. These special patients, in addition to needing our attention for infection prevention and examination bed disinfection, need careful attention to protect them from radiation. For the children who do not cooperate with the examination, family members are required to be present at the examination. These family members also need to receive infection protection and radiation protection. The emergency response budget should also provide for patients with no ability to pay or patients seeking medical treatment from other places. We also provide a pathway to pay fee conveniently as well as free examination services.

In summary, in this war against the COVID-19 epidemic, we have standardized our operations, proceeded with confidence, and come together as a team. We constantly summarize our experience and optimize our workflows (Fig. 1), improving the work efficiency of the team. Every member of the team stays positive in the pursuit of the true human good, as we continue to carry forward the noble goal of medical care and strive for a better and healthier future. During this great epidemic, each of us has upheld a respect for the value of our own lives and limitless care for other lives, which not only has brought individuals and groups together, but also has cultivated a close-knit, mutually shared sense of "community of destiny."

Acknowledgements We thank all our colleagues who helped us during the current study.

\section{Compliance with ethical standards}

Conflict of interest We declare no competing interests.

\section{References}

1. Bellani G, Laffey JG, Pham T, Fan E, Brochard L, Esteban A, et al. Epidemiology, patterns of care, and mortality for patients with acute respiratory distress syndrome in intensive care units in 50 countries. JAMA. 2016;315:788-800.

2. China National Health Commission. Diagnosis and treatment of 2019-nCoV pneumonia in China. In Chinese (Provisional 7th Edition). 2020. http://www.nhc.gov.cn/yzygj/s7652m/202003/a3119 1442e29474b98bfed5579d5af95.shtml. Accessed 6 Mar 2020.

3. Lee EYP, Ng MY, Khong PL. COVID-19 pneumonia: What has CT taught us? Lancet Infect Dis. 2020;20:384-85.

4. Gao J, Tian Z, Yang X. Breakthrough: chloroquine phosphate has shown apparent efficacy in treatment of COVID-19 associated pneumonia in clinical studies. Biosci Trends. 2020;14:72-73.

Publisher's Note Springer Nature remains neutral with regard to jurisdictional claims in published maps and institutional affiliations. 